\title{
TI.117.1
}

\section{TIER Thematic Requirements / Prioritized}

- PDF: TI.117.1-RCrossAvgByRequirement.pdf

- Text: TI.117.1-RCrossAvgByRequirement.txt

\begin{tabular}{|c|c|}
\hline \multicolumn{2}{|c|}{ More Information } \\
\hline Repository ID & TI.117.1 \\
\hline Persistent URL & http://doi.org/10.26869/TI.117.1 \\
\hline Title & TIER Thematic Requirements / Prioritized \\
\hline Authors & Steve Zoppi, Ann West \\
\hline Sponsor & TIER \\
\hline \multicolumn{2}{|l|}{ Review } \\
\hline Status & Legacy \\
\hline Publish Date & April 12, 2016 \\
\hline DOI & 10.26869/TI.117.1 \\
\hline \multicolumn{2}{|l|}{ Signature } \\
\hline Deprecated & No \\
\hline \multicolumn{2}{|l|}{ Future Review } \\
\hline \multicolumn{2}{|l|}{ Supersedes } \\
\hline Format & PDF, Text \\
\hline \multicolumn{2}{|l|}{ Related Docs } \\
\hline \multicolumn{2}{|l|}{ Development Location } \\
\hline \multicolumn{2}{|l|}{ IP Framework } \\
\hline Subject Tags & tier \\
\hline Notes & \\
\hline
\end{tabular}

